\title{
New radiographic classification of endovascular recanalization of symptomatic non-acute carotid artery occlusion
}

\section{Wentao Yan}

Sanbo Brain Hospital, Capital Medical University

\section{Xiuzhen Li}

Sanbo Brain Hospital, Capital Medical University

\section{Wei Guo}

Sanbo Brain Hospital, Capital Medical University

Jiachun Liu ( $\nabla$ jiachunliu2003@mail.ccmu.edu.cn )

Sanbo Brain Hospital, Capital Medical University https://orcid.org/0000-0002-7797-8969

\section{Changxiang Yan}

Sanbo Medical University, Capital Medical University

\section{Research Article}

Keywords: Non-acute carotid occlusion, cerebral angiography, carotid occlusion classification, prognosis, recurrence.

Posted Date: March 7th, 2022

DOl: https://doi.org/10.21203/rs.3.rs-962676/v1

License: (1) This work is licensed under a Creative Commons Attribution 4.0 International License. Read Full License 


\section{Abstract}

Purpose: To propose a new imaging classification based on cerebral angiography to determine the segment and length of occlusion and the condition of the proximal and distal lumen of the occluded segment.

Methods: This retrospective study included patients with non-acute carotid artery occlusion (NACAO) who visited XXX Hospital (01/2017-12/2020). The lesions were classified according to the segment and length of carotid artery occlusion and the proximal and distal lumen of the occluded segment.

Results: Type IV is not recommended for endovascular recanalization, and patients with types I (10/24, $41.7 \%)$, II (7/24, 29.2\%), and III (7/24, 29.2\%) were included. The recanalization success rate was $87.5 \%$ (21/24; Type I, 10/10; Type II, 7/7; Type III, 4/7; $P=0.017$ ). The incidence of perioperative complications was 25.0\% (6/24; Type I, 0/10; Type II, 2/7; Type III, 4/7; P=0.038). The re-occlusion rate was not significantly different among the three groups $(P=0.284)$. The average modified Rankin Scale (mRS) score in the success group was lower than before operation $(P=0.012)$, but not in the failure group $(P=0.317)$.

Conclusion: The new classification based on cerebral angiography is helpful to screen patients with drugrefractory NACAO with a high success rate and low complications of intra-tube recanalization.

\section{Introduction}

Carotid artery stenosis is a narrowing of the carotid artery due to atherosclerosis and is associated with an increased risk of transient ischemic attacks and stroke $[1,2,3]$. The estimated prevalence of asymptomatic carotid artery stenosis ( $\geq 50 \%$ stenosis) is $<10 \%$ in the general population but higher in patients $>65$ years old, patients with coronary artery or peripheral vascular disease, and in patients who have risk factors for cardiovascular disease $[3,4]$. The prognosis of symptomatic carotid artery occlusion (CAO) is generally poor, with $70 \%$ of the cases with early neurological recurrence [5] or recurrent stroke within 2 weeks [6].

After an acute anterior circulation ischemic stroke caused by CAO, the timely opening of the occluded vessels within the treatment time window can significantly improve patients' prognosis. Drugs are mainly used to treat and control the risk factors for patients with non-acute CAO (NACAO) beyond the time window. Nevertheless, even under strict drug therapy, insufficient perfusion in the CAO supply area can still cause stroke recurrence, cognitive decline, and poor functional prognosis $[1,7]$. The shedding of the embolus at the occluded site can also lead to stroke recurrence.

Endovascular treatment is now possible to achieve a normal physiological forward blood flow $[8,9,10$, 11]. Screening patients with a high success rate with vascular recanalization through imaging examination before the operation is important to undertake as early as possible the treatment with the highest likelihood of improving the patient's condition and minimizing the long-term risk of recurrence 
and complications. Lee et al. [12] classified the patients with symptomatic CAO according to the side of involvement relative to the clinoid segment based on CTA. Lee et al. [12] showed that the rates of technical success, complications, and re-occlusion were $52 \%, 22 \%$, and $91 \%$ for group A (occlusion at or distal to the clinoid segment) and $89 \%, 0 \%$, and $0 \%$ for group B (occlusion proximal to the clinoid segment). Hasan et al. [13] classified the patients according to the shape of the occluded proximal stump and the blood flow filling site of the occluded distal stump. In Hasan et al. [13], the success/complications rates were $100 \% / 0 \%$ for group $A, 100 \% / 13 \%$ for group $B, 50 \% / 38 \%$ for group $C$, and $25 \% / 25 \%$ for group D. Mo et al. [14] improved the classification by Hasan et al. [13]. In Mo et al. [14], the success rates were $90 \%$ for group A, $70 \%$ for group B, $91 \%$ for group C, and $0 \%$ for group $D$. Nevertheless, these methods all have some shortcomings. The boundary between different types is not obvious, or there is no significant difference in vascular recanalization rates and complications among different types. In addition, the few available reports lack a unified inclusion standard, and the success rates of recanalization and perioperative complications are quite different $[12,13,15,16]$. Therefore, it is important to propose a more rational classification method that has an actual prognostic value.

The present study proposed a new imaging classification based on cerebral angiography to determine the segment and length of occlusion and the condition of the proximal and distal lumen of the occluded segment and evaluated this classification on the success rate of endovascular recanalization, perioperative complications, and long-term re-occlusion in patients with NACAO.

\section{Materials And Methods}

\section{Study design and patients}

This retrospective study included patients with NACAO who visited the Neuro-interventional Department of XXX Hospital from January 2017 to December 2020. The diagnosis was based on carotid ultrasound, magnetic resonance angiography (MRA), and/or computed tomography angiography (CTA), showing no blood flow through the occluded segment of the carotid artery. The inclusion criteria were 1) 18-75 years of age, 2) Carotid ultrasound, CTA, and/or MRA confirmed unilateral or bilateral CAO (no blood flow signal in the lumen), and 3) the occluded site included the common carotid artery (CCA) or internal carotid artery (ICA). The exclusion criteria were: 1 ) severe disability (modified Rankin Scale (mRS) $\geq 4$ ), 2) accompanied by severe complications such as severe cardiopulmonary insufficiency or malignant tumor, 3 ) intracranial aneurysms or cerebral arteriovenous malformations, 4) peptic tract ulcer or other bleeding diseases, 5) contrast agent allergy or renal insufficiency that could not tolerate contrast, or 6) pregnancy or perinatal period. The procedures followed were in accordance with the "Declaration of Helsinki" and the ethical standards of the responsible committee on human experimentation (the Ethics Committee of our hospital). This study was approved by the Ethics Committee (SBNK-YJ-2021-005-01) of our hospital. The requirement for informed consent was waived by the committee.

\section{Classification of carotid artery occlusion}


Based on many years of clinical experience in NACAO endovascular recanalization and repeated and indepth study of patients' DSA image data, CAO is divided into the following four types according to the segment, length, and whether the proximal and distal lumens are developed. The ICA segmentation was based on the seven-segment method proposed by Bouthillier ( $C 1$, cervical; $C 2$, petrous; $C 3$, lacerum; $C 4$, cavernous; C5, clinoidal; C6, ophthalmic; and C7, communicating) [17]. In type I, the occluded segment is located in the CCA or involves $\leq 2$ consecutive segments in C1-C7. In type II, the occluded segment involves $\geq 3$ consecutive internal carotid artery segments proximal to C5. In type III, the occluded segment involves $\geq 3$ consecutive ICA segments, including C5. In type IV, the occluded proximal or distal lumen of the carotid artery is completely unrecognizable (Figure 1). [17] ${ }^{17}$

\section{Vascular recanalization therapy}

Patients with CAO meeting the following indications and contraindications were suitable for endovascular recanalization. The indications were a) color Doppler ultrasound, CTA, and/or MRA showed occlusion of the CCA or ICA and was confirmed by cerebral angiography to have no forward blood flow at all, b) ischemic symptoms consistent with the occlusion side, and ischemic events still occurred under the conditions of anti-platelet aggregation, statins, and strict control of risk factors, c) computed tomographic perfusion (CTP) or perfusion-weighted imaging (PWI) confirmed that there was an obvious decrease in cerebral blood flow around the infarcted area within the CAO blood supply range, and d) the Alberta Stroke Program Early Computerized Tomography Score (ASPECT) score of the occluded side was $\geq 6$ points [18]. The contraindications were a) asymptomatic or stable CAO after drug treatment, b) acute CAO within the time window of mechanical thrombectomy, c) CAO with anterior blood flow of grade 1, d) history of stent implantation in the CAO site, e) the proximal or distal lumen of CAO cannot be identified at all, i.e., type IV CAO, f) high-resolution magnetic resonance imaging showed obvious tortuosity of blood vessels in the occluded segment, or g) renal failure or severe allergy to contrast agents.

All patients underwent magnetic resonance imaging (MRI)/diffusion-weighted imaging (DWI), highresolution MRI, PWI, or CTP, and digital subtraction angiography (DSA) before operation. Aspirin entericcoated tablets ( $100 \mathrm{mg}$, once a day) and clopidogrel tablets (75 mg, once a day) were taken regularly for at least 5 days before the operation. The operation was performed by endotracheal intubation and general intravenous anesthesia (sufentanil, propofol, and rocuronium). Bilateral femoral arteries were punctured and placed with vascular sheaths, $8 \mathrm{~F}$ on the right side and $4 \mathrm{~F}$ on the left side. Whole-body heparinization ( $80 \mathrm{U} / \mathrm{kg}$ ) was performed, and activated clotting time (ACT) was monitored. An 8F guide catheter (Mach1, Boston Scientific, Natick, MA, USA) was placed at the proximal end of the occlusion, and a proximal balloon occlusion catheter (Merci, Concentric Medical, Stryker Corp., Mountain View, CA, USA) was used if there was sufficient space at the proximal end. A 4F catheter (VER, Cordis Medical, Fremont, CA, USA) was placed in the contralateral ICA, vertebral artery, or ipsilateral external carotid artery to show occluded distal lumen (i.e., the collateral branch formed by the contralateral ICA, vertebral artery, or ipsilateral external carotid artery). Simultaneous double catheter angiography showed the proximal and distal lumen of the occluded segment and served as a road map to guide endovascular recanalization. A microcatheter (Excelsior SL-10, Stryker; Echelon-10, ev3 Endovascular Inc., Plymouth, MN, USA) combined 
with a microwire (PILOT 150, Abbott; PT2, Boston Scientific, Natick, MA, USA) was used to try to break through the proximal end of the occlusion. If it failed, a 4F contrast catheter (MP A1 $125 \mathrm{~cm}$, Cordis Medical, Fremont, CA, USA) combined with a super slippery guidewire (Terumo, Tokyo, Japan) was used to break through the proximal end of the occlusion. After the microguidewire and microcatheter passed through the occluded segment, the angiography confirmed that they were in the normal lumen, and then the exchange guidewire (Synchro, Stryker Corp., Kalamazoo, MI, USA) was placed in the distal normal lumen. If the occlusion's distal end was located proximal to C2, a distal protection device (Spider FX, ev3 Endovascular Inc., Plymouth, MN, USA) was used. The proximal balloon occlusion catheter was filled to block the flow. A small balloon (Ryujin, Terumo, Tokyo, Japan) with a diameter of $2 \mathrm{~mm}$ was used to expand from far to near with a microguidewire as the track, and the lumen was judged by angiography. The sacculus expansion stent (Apollo, MicroPort Scientific Corp., Shanghai, China) with a 3-4 mm diameter was placed at sites with irregularities or obvious stenosis at the site distal to $\mathrm{C} 1$. The proximal part of C1 was reconstructed with a self-expanding stent (Wallstent, Boston Scientific, Natick, MA, USA). A sacculus (Ryujin, Terumo, Tokyo, Japan; Sterling, Boston Scientific, Natick, MA, USA) with a 2-5 mm diameter was used for posterior dilation when the residual stenosis was more than $30 \%$ confirmed by angiography. Finally, ipsilateral carotid angiography was performed to evaluate the anterior blood flow according to the thrombolysis in the cerebral infarction ( $\mathrm{TICl}$ ) grading system [19]. TICI $2 \mathrm{~b}$ or 3 levels are defined as successful recanalization.

\section{Postoperative care}

ECG and blood pressure monitoring were carried out after the operation. The systolic blood pressure was strictly controlled to be lower than the baseline pressure by $10-20 \mathrm{mmHg}$ within 24 days after the operation. Nicardipine was injected intravenously when blood pressure was high, and dopamine was injected intravenously when blood pressure was low due to carotid sinus reflex. Patients' consciousness, speech, limb activities, and other neurological functional states were monitored. If there was any abnormality, carotid vascular ultrasound, brain CT, and/or magnetic resonance imaging were performed to timely find and address complications such as stent thrombosis, cerebral hemorrhage, and cerebral infarction.

\section{Observation indicators}

The differences in the success rate of endovascular recanalization, the incidence of re-occlusion, and the incidence of complications were observed among different types of patients. In addition, the long-term prognosis of the patients of different types was compared.

\section{Follow-up}

Clinical follow-up was performed routinely at 1, 3, 6, and 12 months after the operation. The mRS score and whether stroke or transient ischemic attack (TIA) occurred were followed once a year. Color Doppler ultrasound of cervical vessels was reexamined 1 week and 1 month after the operation. CTA or DSA was reexamined 3-12 months after the operation, and imaging reexamination was performed at any time if 
stroke or TIA occurred. Images showing stenosis of $>50 \%$ of the lumen after recanalization were defined as restenosis, and no forward blood flow in the lumen was defined as re-occlusion.

\section{Statistical analysis}

SPSS 22.0 (IBM Corp, Armonk, NY, USA) was used to analyze the data. The Kolmogorov-Smirnov test was used to test whether the continuous data were normally distributed. Continuous data with a normal distribution are expressed as means \pm standard deviations and were analyzed using the paired t-test (within-group comparisons) or ANOVA with Tukey's post hoc test (multiple group comparison).

Continuous data with a non-normal distribution are presented as medians ( $25^{\text {th }}-75^{\text {th }}$ quartiles) and were analyzed using the Wilcoxon symbolic rank test (within-group comparisons) or the Kruskal-Wallis test for multiple comparisons. The categorical data are expressed as $\mathrm{n}(\%)$ and were analyzed using Fisher's exact test. Two-sided P-values $<0.05$ were considered statistically significant.

\section{Results}

\section{Characteristics of the patients}

From January 2017 to December 2020, 24 NACAO of 23 patients were treated with endovascular recanalization, among which one patient underwent bilateral CCA occlusion recanalization because of multiple cervical radiotherapy sessions after esophageal cancer resection. The characteristics, treatment, and follow-up characteristics of the patients are shown in Table 1 and Supplementary Table S1.

Seventeen patients $(17 / 23,73.9 \%)$ were male. The mean age was $59.3 \pm 10.8$ years. Recurrent symptoms before surgery included stroke $(15 / 23,65.2 \%), \operatorname{TIA}(7 / 23,30.4 \%)$, and amaurosis $(1 / 23,4.3 \%)$. Thirteen lesions $(13 / 24,54.2 \%)$ were located on the left side. The median time from occlusion to recanalization was 34.5 (19.0-64.5) days. Occlusion types included type I (10/24, 41.7\%), type II (7/24, 29.2\%), and type III $(7 / 24,29.2 \%)$. Five patients $(5 / 22,22.7 \%)$ underwent contralateral carotid artery stenting, and six patients $(6 / 23,26.1 \%)$ underwent vertebrobasilar artery or subclavian artery stenting. The main collateral circulation included anterior communicating artery $(19 / 24,79.2 \%)$, posterior communicating artery $(10 / 24,41.7 \%)$, and ophthalmic artery (13/24, 54.2\%). 
Table 1

Baseline characteristics of the patients and occluded carotid artery

\begin{tabular}{|c|c|c|c|c|}
\hline \multirow[t]{2}{*}{ Characteristics } & \multicolumn{3}{|c|}{ Patients $(\mathrm{N}=23)$} & \multirow[t]{2}{*}{$\mathbf{P}$} \\
\hline & $I(n=9)$ & II (n=7) & III $(n=7)$ & \\
\hline Age (years) & $56.8 \pm 15.2$ & $64.4 \pm 6.0$ & $57.3 \pm 6.3$ & 0.330 \\
\hline Male sex & $5(55.6)$ & $7(100.0)$ & $5(71.4)$ & 0.173 \\
\hline \multicolumn{5}{|l|}{ Baseline medical history, n (\%) } \\
\hline Hypertension & $3(33.3)$ & $5(71.4)$ & $2(28.6)$ & 0.323 \\
\hline Diabetes mellitus & $4(44.4)$ & $3(42.9)$ & $2(28.6)$ & 0.874 \\
\hline Hyperlipidemia & $3(33.3)$ & $6(85.7)$ & $4(57.1)$ & 0.126 \\
\hline Smoking & $5(55.6)$ & $5(71.4)$ & $4(57.1)$ & 0.874 \\
\hline Coronary artery disease & $2(22.2)$ & $1(14.3)$ & 3 (42.9) & 0.598 \\
\hline Atrial fibrillation & 0 & 0 & $1(14.3)$ & 0.609 \\
\hline Prior neck radiotherapy & $1(11.1)$ & 0 & 0 & $>0.999$ \\
\hline Symptoms, n (\%) & & & & 0.450 \\
\hline Recurrent TIA & $4(44.4)$ & $1(14.3)$ & $2(28.6)$ & \\
\hline Recurrent stroke & $4(44.4)$ & $6(85.7)$ & $5(71.4)$ & \\
\hline Recurrent amaurosis fugax & $1(11.1)$ & 0 & 0 & \\
\hline Preoperative NIHSS, median (IQR) & $0(0-8.5)$ & $6(1-10)$ & $4(1-6)$ & 0.379 \\
\hline Preoperative mRS, median (IQR) & $1(1-4)$ & $2(1-4)$ & $3(2-3)$ & 0.770 \\
\hline \multirow[t]{2}{*}{ Characteristics } & \multicolumn{3}{|c|}{ Carotid artery $(n=24)$} & $\mathbf{P}$ \\
\hline & $I(n=10)$ & II (n=7) & III $(n=7)$ & \\
\hline Recanalization side of CAO, $n(\%)$ & & & & 0.522 \\
\hline Left & $4(40)$ & $4(57.1)$ & $5(71.4)$ & \\
\hline Right & $6(60)$ & $3(42.9)$ & $2(28.6)$ & \\
\hline \multicolumn{5}{|c|}{$\begin{array}{l}\text { Time to recanalization (days) median } \\
\text { (IQR) }\end{array}$} \\
\hline \multicolumn{5}{|c|}{$\begin{array}{l}\text { The Continuous data with a normal distribution is expressed as means } \pm \text { standard deviations. The } \\
\text { categorical data are expressed in n (\%). TIA: transient ischemic attack; NIHSS: National Institutes of } \\
\text { Health Stroke Scale; IQR: interquartile range; mRS: modified Rankin Scale; CAO: carotid artery } \\
\text { occlusion; CA: carotid artery; VBA: vertebrobasilar artery; SCA: subclavian artery; ASITN/SIR: American } \\
\text { Society of Interventional Therapeutic Neuroradiology/Society of Interventional Radiology; ASPECT: } \\
\text { Alberta Stroke Program Early Computerized Tomography Score. }\end{array}$} \\
\hline
\end{tabular}




\begin{tabular}{|c|c|c|c|c|}
\hline \multirow[t]{2}{*}{ Characteristics } & \multicolumn{3}{|c|}{ Patients $(\mathrm{N}=23)$} & \multirow[t]{2}{*}{$\mathbf{P}$} \\
\hline & I (n=9) & II (n=7) & III $(n=7)$ & \\
\hline Initial symptom to recanalization & $\begin{array}{l}68.5(46.8- \\
125.0)\end{array}$ & $\begin{array}{l}61.0(38.0- \\
80.0)\end{array}$ & $\begin{array}{l}70.0(18.0- \\
90.0)\end{array}$ & 0.780 \\
\hline $\begin{array}{l}\text { Radiological occlusion to } \\
\text { recanalization }\end{array}$ & $\begin{array}{l}33.0(19.0- \\
45.3)\end{array}$ & $\begin{array}{l}54.0(19.0- \\
79.0)\end{array}$ & $\begin{array}{l}40.0(14.0- \\
69.0)\end{array}$ & 0.456 \\
\hline Last symptom to recanalization & $\begin{array}{l}25.0(14.5- \\
34.5)\end{array}$ & $\begin{array}{l}23.0(17.0- \\
31.0)\end{array}$ & $\begin{array}{l}17.0(10.0- \\
36.6)\end{array}$ & 0.668 \\
\hline Stenting of contralateral CA, n (\%) & $3(30.0)$ & $2(28.6)$ & 0 & 0.447 \\
\hline Stenting of VBA or SCA, $n$ (\%) & $2(20.0)$ & $3(42.9)$ & $1(14.3)$ & 0.590 \\
\hline \multicolumn{5}{|l|}{ Collateral circulation, n (\%) } \\
\hline Anterior communicating artery & $8(80.0)$ & $5(71.4)$ & $6(85.7)$ & $>0.999$ \\
\hline Posterior communicating artery & $2(20.0)$ & $4(57.1)$ & $4(57.1)$ & 0.262 \\
\hline Ophthalmic artery & $3(30.0)$ & $5(71.4)$ & $5(71.4)$ & 0.194 \\
\hline $\begin{array}{l}\text { Preoperative ASITN/SIR, median } \\
\text { (IQR) }\end{array}$ & $3(3-4)$ & $3(3-3)$ & $3(3-3)$ & 0.072 \\
\hline Preoperative ASPECT, median (IQR) & $8(7.8-10)$ & 7 (7-9) & $10(7-10)$ & 0.396 \\
\hline \multicolumn{5}{|c|}{$\begin{array}{l}\text { The Continuous data with a normal distribution is expressed as means } \pm \text { standard deviations. The } \\
\text { categorical data are expressed in n (\%). TIA: transient ischemic attack; NIHSS: National Institutes of } \\
\text { Health Stroke Scale; IQR: interquartile range; mRS: modified Rankin Scale; CAO: carotid artery } \\
\text { occlusion; CA: carotid artery; VBA: vertebrobasilar artery; SCA: subclavian artery; ASITN/SIR: American } \\
\text { Society of Interventional Therapeutic Neuroradiology/Society of Interventional Radiology; ASPECT: } \\
\text { Alberta Stroke Program Early Computerized Tomography Score. }\end{array}$} \\
\hline
\end{tabular}

\section{Success rate of endovascular recanalization in different types of lesions}

The microwire successfully passed through 23 (23/24, 95.8\%) lesions, and 21 occluded arteries (21/24, $87.5 \%$ ) were successfully re-opened. The success rate in type I was $100 \%(10 / 10)$ (Figure 2, a-i), $100 \%$ in type II (7/7) (Fig. 3, a-d), and 57.1\% in type III (4/7) (Figure 3, e-h). Complications occurred in six patients $(6 / 24,25.0 \%)$ during the perioperative period. Type I had no complications. Complications occurred in two patients $(2 / 7,28.6 \%)$ with type II lesions, including one case of contrast-induced encephalopathy with transient cortical blindness and one case of cerebral hyperperfusion syndrome with headache and hyperactivity. Complications occurred in four cases $(4 / 7,57.1 \%)$ with type III lesions, including two cases of distal embolization, carotid-cavernous fistula in one case, and one case of acute in-stent thrombosis. The complications recovered completely in a short time after treatment in five patients, and permanent neurological dysfunction was caused by a perioperative stroke caused by distal embolism in one patient. The success rate of recanalization $(P=0.017)$ and the incidence of complications $(P=0.038)$ were significantly different among the three types of lesions (Table 2). 
Table 2

Procedure success, complications, and re-occlusion among types of CAO

\begin{tabular}{|lllll|}
\hline Characteristics & Total & \multicolumn{2}{c|}{ Type of CAO } & P \\
\cline { 2 - 4 } & & I+I & III & \\
\cline { 1 - 4 } Procedure success & $21(24)$ & $17(17)$ & $4(7)$ & 0.017 \\
\cline { 2 - 4 } & $87.5 \%$ & $100.0 \%$ & $57.1 \%$ & \\
\hline Complication & $6(24)$ & $2(17)$ & $4(7)$ & 0.038 \\
\cline { 2 - 4 } & $25.0 \%$ & $11.8 \%$ & $57.1 \%$ & \\
\hline Reocclusion & $2(20)$ & $1(17)$ & $1(3)$ & 0.284 \\
\cline { 2 - 4 } & $10.0 \%$ & $5.9 \%$ & $33.3 \%$ & \\
\hline The categorical data are expressed in n (\%). & \\
\hline
\end{tabular}

\section{Prognosis}

All 23 patients $(23 / 23,100 \%)$ were followed for a median time of 17 (3-41) months. Fourteen patients $(14 / 23,60.9 \%)$ had mRS scores of $\leq 2,13(13 / 23,46.5 \%)$ had mRS scores lower than before the operation, eight $(8 / 23,34.8 \%)$ had $m R S$ scores consistent with before operation, and two $(2 / 23,8.7 \%)$ had $\mathrm{mRS}$ scores higher than before the operation. In the success group (20 patients), the average mRS score during follow-up was lower than before operation $(Z=-2.524, P=0.012)$. There were no significant differences between the average follow-up mRS score and the average preoperative $\mathrm{mRS}$ score in the failure group (three patients) $(Z=-1.000, P=0.317)$ (Figure 4). During follow-up, stroke occurred in two patients $(2 / 23,8.7 \%)$, one patient had a re-occlusion (Figure $3, \mathrm{e}-\mathrm{h})$, and one case has a failed recanalization. A total of $20(20 / 21,95.2 \%)$ carotid arteries were successfully recanalized and were followed by imaging, with a median imaging follow-up of 3 months. Among them, two patients $(2 / 20$, $10 \%)$ had restenosis, and all of them underwent secondary stenting. Re-occlusion occurred in two patients $(2 / 20,10 \%)$, including one case of type II and one case of type III. There were no significant differences in the re-occlusion rates among types I, II, and III ( $P=0.284)$ (Table 2).

\section{Discussion}

The available classifications of CAO lack a unified inclusion standard, and the success rates of recanalization and perioperative complications are different $[12,13,15,16]$. Therefore, this study aimed to propose a new imaging classification based on cerebral angiography to determine the segment and length of occlusion and the condition of the proximal and distal lumen of the occluded segment and to evaluate this classification on the success rate of endovascular recanalization, perioperative complications, and long-term re-occlusion in patients with NACAO. The results suggest that the new 
classification based on cerebral angiography is helpful to screen patients with drug-refractory NACAO with a high success rate and low complications of intra-tube recanalization. Type I and type II might be more suitable for endovascular recanalization.

Even after strict drug treatment and control of risk factors, NACAO can still lead to stroke and cognitive decline [7]. The intracranial and extracranial bypasses for CAO cannot effectively reduce long-term stroke occurrence [20]. With the development of neurointerventional technology and materials, many studies have preliminarily shown that it is technically feasible to perform endovascular recanalization for NACAO, restoring forward physiological blood flow, reducing the occurrence of long-term stroke, improving cerebral perfusion, and improving cognitive function $[1,16,21]$. Still, endovascular recanalization for NACAO is difficult, and there are many complications. It is important to screen patients to identify those with an expected high technical success rate and low occurrence of complications. Based on the summary of 119 cases of NACAO imaging data, especially cerebral angiography, and compared with the literature, this study proposed a new NACAO classification, which can include all clinical NACAO conditions and has certain guiding significance for endovascular recanalization. According to the segment and length of CAO and the proximal and distal lumen of the occluded segment, the patients could be divided into four types. Type IV was not recommended for endovascular recanalization. The overall success rate of endovascular recanalization in the other three types was $87.5 \%$, and the success rate of type I and type II was higher than that of type III. The overall complication rate was $25.0 \%$, and all the complications recovered completely in a short time except for one case. The complication rates of types I and II were lower than those of type III.

$[7]^{7}$ The cornerstone of successful endovascular canalization for NACAO is to break through the beginning of the occlusion and enter the true lumen or subintima, then pass through the occluded segment and enter the true lumen. An identifiable proximal lumen that can provide sufficient support for the guidewire and catheter is the basic requirement for canalization. If there is no identifiable proximal lumen, endovascular recanalization alone is difficult to be achieved. This situation was classified as type IV and hence is not recommended for endovascular recanalization. In such cases, carotid endarterectomy can be performed first, followed by endovascular recanalization (hybrid surgery), but this will be examined in future studies.

Type I in this classification is characterized by short, occluded segments and relatively straight occlusion segments, easy passage of the microwires, microcatheters, balloons, and stents, high technical success rate and low complications, short stents, and low long-term restenosis or re-occlusion rate. The difference between types II and III and type I is that the involved segments are longer in types II and III. The difference between type III and type II is that the clinoid process segment and its proximal and distal segments are involved. The siphon part of the carotid artery, especially the clinoid process segment and its proximal and distal ends, is obviously tortuous and angled. It is the most difficult part for interventional equipment to pass through the ICA even if the lumen is not occluded. When the occluded segment involves the clinoid process segment and its proximal and distal ends, the catheter and guidewire need to pass through the occluded segment at a larger angle or even in the opposite direction. 
At this moment, the component force consistent with the direction of the blood vessel is small, and it is not easy to pass through the occluded segment. In contrast, the component force perpendicular to the direction of the blood vessel is large, and it is easy to cause dissection or even perforation of the artery. The present study showed that compared with type I and type II, type III lesions had a lower success rate of endovascular recanalization and more complications.

Lee et al. [12] divided NACAO into two groups according to CTA. Group A involved the clinoid process segment and the distal artery, while Group B involved the proximal part of the clinoid process segment or CCA. The technical success rate of Group B was higher than that of Group A, while the incidence of complications and long-term re-occlusion rates were lower than in Group A. These results support those of the present study, but the present study proposes a refinement of their Group B, which includes types I and II of the present study. Long occlusion segment and occlusion involving clinoid process segment have a lower success rate, higher complication rate, and long-term re-occlusion rate [12]. Although the classification is simple, it has certain limitations. For example, the simple short and straight ocular segment occlusion and communicating segment occlusion, which is easy to open, are classified into Group A, which is difficult to open. At the same time, Group B is too general to distinguish short from long-segment occlusion. In addition, the classification does not consider the proximal end of the occlusion, and breaking through the proximal end is the beginning and very important link of occlusion reopening.

Hasan et al. [13] classified ICA occlusion into four types (A, B, C, and D) based on cerebral angiography according to the shape and position of the occlusion's proximal end and the segment of blood flow reconstruction at the distal end of the occlusion. The common point of types $A, B$, and $C$ is that the distal cavernous sinus segment or petrous segment is filled with blood flow. The main difference among the three types lies in the proximal end of the occlusion. The proximal lumen of type $A$ is tapered, the proximal lumen of type $B$ is not tapered, and the proximal lumen of type $C$ has no identifiable proximal lumen. Type $D$ is that there is no recognizable proximal lumen, and there is no blood flow filling in the distal cavernous sinus segment and petrous segment. This classification does not include cases where there is an identifiable lumen at the proximal end, there is no blood flow filling in the distal cavernous sinus segment and petrous segment, and simple ocular segment or communicating segment occlusion $[13,14]$. In addition, there is no essential difference between types $A$ and $B$. The key point of the proximal end is to have an identifiable lumen and provide sufficient support. There is no significant difference in recanalization success rate and complication rate between the two.

Hence, the classification suggested here might have advantages over previous classifications. Still, additional studies are necessary to confirm and refine the results. In addition, there are some limitations in this study. Due to the strict inclusion criteria, the number of patients was small, and the comparison among the three types was limited. Types I and II were combined for comparison with type III, but type I might be easier to recanalize than type II. Besides, the study had no control group and could not examine whether endovascular recanalization of NACAO has more advantages than drug therapy in preventing stroke. This study was a retrospective study, with the possibility of selection bias and recall bias, and the 
level of evidence is low. Future prospective studies should examine endovascular recanalization therapy for types I and II NACAO. This study was a single-center study, and the results cannot be generalized.

\section{Conclusion}

This study proposes a new classification of NACAO based on the segmental involvement of NACAO and the condition of the proximal and distal lumen of the occluded segment. Types I and II have a higher success rate of endovascular recanalization and lower incidence of complications than type III. Type IV is not recommended for endovascular recanalization. Endovascular recanalization for NACAO with poor drug treatment effect is technically feasible and with a low complication rate after a strict patient selection, which might improve the long-term prognosis to a certain extent.

\section{Declarations}

\section{Funding}

This research was supported by the project of the Beijing Municipal Science \& Technology Commission (Z171100000417046).

\section{Conflicts of interest}

No conflict of interest.

\section{Availability of data and material}

The datasets used and/or analyzed during the current study are available from the corresponding author on reasonable request.

\section{Code availability}

Not applicable.

\section{Ethics approval}

The procedures followed were in accordance with the "Declaration of Helsinki" and the ethical standards of the responsible committee on human experimentation (the Ethics Committee of Sanbo Brain Hospital of Capital Medical University). This study was approved by the Ethics Committee (SBNK-YJ-2021-005-01) of Sanbo Brain Hospital of Capital Medical University.

\section{Consent to participate}

The requirement for informed consent was waived by the committee.

\section{Acknowledgments}


We would like to thank our colleagues Wang Yabin (for her help in recording and sorting out clinical data) and Zhang Xiaofeng (for her help in sorting out the image data).

\section{References}

1. Brott TG, Halperin JL, Abbara S, Bacharach JM, Barr JD, Bush RL, Cates CU, Creager MA, Fowler SB, Friday G, Hertzberg VS, Mclff EB, Moore WS, Panagos PD, Riles TS, Rosenwasser RH, Taylor AJ, American College of Cardiology Foundation/American Heart Association Task Force on Practice G. American Stroke A, American Association of Neuroscience N, American Association of Neurological $\mathrm{S}$, American College of R, American Society of $\mathrm{N}$, Congress of Neurological S, Society of Atherosclerosis I, Prevention, Society for Cardiovascular A, Interventions, Society of Interventional R, Society of Neurolnterventional S, Society for Vascular M, Society for Vascular S. American Academy of N, Society of Cardiovascular Computed T. (2011) 2011

ASA/ACCF/AHA/AANN/AANS/ACR/ASNR/CNS/SAIP/SCAI/SIR/SNIS/SVM/SVS guideline on the management of patients with extracranial carotid and vertebral artery disease: executive summary. Stroke 42:e420-463. 10.1161/STR.0b013e3182112d08.

2. Grotta JC. Clinical practice. Carotid stenosis. N Engl J Med. 2013;369:1143-50. 10.1056/NEJMcp1214999.

3. Thapar A, Jenkins IH, Mehta A, Davies AH. Diagnosis and management of carotid atherosclerosis. BMJ. 2013;346:f1485. 10.1136/bmj.f1485.

4. Taussky P, Hanel RA, Meyer FB. Clinical considerations in the management of asymptomatic carotid artery stenosis. Neurosurg Focus. 2011;31:E7. 10.3171/2011.9.FOCUS11222.

5. Ois A, Cuadrado-Godia E, Rodriguez-Campello A, Jimenez-Conde J, Roquer J. High risk of early neurological recurrence in symptomatic carotid stenosis. Stroke. 2009;40:2727-31.

10.1161/STROKEAHA.109.548032.

6. Marnane M, Ni Chroinin D, Callaly E, Sheehan OC, Merwick A, Hannon N, Horgan G, Kyne L, Moroney J, McCormack PM, Dolan E, Duggan J, Williams D, Crispino-O'Connell G, Kelly PJ. Stroke recurrence within the time window recommended for carotid endarterectomy. Neurology. 2011;77:738-43. 10.1212/WNL.0b013e31822b00cf.

7. Flaherty ML, Flemming KD, McClelland R, Jorgensen NW, Brown RD Jr. Population-based study of symptomatic internal carotid artery occlusion: incidence and long-term follow-up. Stroke. 2004;35:e349-52. 10.1161/01.STR.0000135024.54608.3f.

8. Abbott AL, Paraskevas KI, Kakkos SK, Golledge J, Eckstein HH, Diaz-Sandoval LJ, Cao L, Fu Q, Wijeratne T, Leung TW, Montero-Baker M, Lee BC, Pircher S, Bosch M, Dennekamp M, Ringleb P. Systematic Review of Guidelines for the Management of Asymptomatic and Symptomatic Carotid Stenosis. Stroke. 2015;46:3288-301. 10.1161/STROKEAHA.115.003390. 
9. Kernan WN, Ovbiagele B, Black HR, Bravata DM, Chimowitz MI, Ezekowitz MD, Fang MC, Fisher M, Furie KL, Heck DV, Johnston SC, Kasner SE, Kittner SJ, Mitchell PH, Rich MW, Richardson D, Schwamm LH, Wilson JA, American Heart Association Stroke Council CoC, Stroke Nursing CoCC, Council on Peripheral Vascular D. Guidelines for the prevention of stroke in patients with stroke and transient ischemic attack: a guideline for healthcare professionals from the American Heart Association/American Stroke Association. Stroke. 2014;45:2160-236.

10.1161/STR.0000000000000024.

10. Powers WJ, Rabinstein AA, Ackerson T, Adeoye OM, Bambakidis NC, Becker K, Biller J, Brown M, Demaerschalk BM, Hoh B, Jauch EC, Kidwell CS, Leslie-Mazwi TM, Ovbiagele B, Scott PA, Sheth KN, Southerland AM, Summers DV, Tirschwell DL. Guidelines for the Early Management of Patients With Acute Ischemic Stroke: 2019 Update to the 2018 Guidelines for the Early Management of Acute Ischemic Stroke: A Guideline for Healthcare Professionals From the American Heart Association/American Stroke Association. Stroke. 2019;50:e344-418. 10.1161/STR.0000000000000211.

11. Wein T, Lindsay MP, Cote R, Foley N, Berlingieri J, Bhogal S, Bourgoin A, Buck BH, Cox J, Davidson D, Dowlatshahi D, Douketis J, Falconer J, Field T, Gioia L, Gubitz G, Habert J, Jaspers S, Lum C, McNamara Morse D, Pageau P, Rafay M, Rodgerson A, Semchuk B, Sharma M, Shoamanesh A, Tamayo A, Smitko E, Gladstone DJ, Heart, Stroke Foundation Canadian Stroke Best Practice C. Canadian stroke best practice recommendations: Secondary prevention of stroke, sixth edition practice guidelines, update 2017. Int J Stroke. 2018;13:420-43. 10.1177/1747493017743062.

12. Lee CW, Lin YH, Liu HM, Wang YF, Chen YF, Wang JL. Predicting procedure successful rate and 1-year patency after endovascular recanalization for chronic carotid artery occlusion by CT angiography. Int J Cardiol. 2016;221:772-6. 10.1016/j.ijcard.2016.07.127.

13. Hasan D, Zanaty M, Starke RM, Atallah E, Chalouhi N, Jabbour P, Singla A, Guerrero WR, Nakagawa D, Samaniego EA, Mbabuike N, Tawk RG, Siddiqui AH, Levy El, Novakovic RL, White J, Schirmer CM, Brott TG, Shallwani H, Hopkins LN. (2018) Feasibility, safety, and changes in systolic blood pressure associated with endovascular revascularization of symptomatic and chronically occluded cervical internal carotid artery using a newly suggested radiographic classification of chronically occluded cervical internal carotid artery: pilot study. J Neurosurg:1-10. 10.3171/2018.1.JNS172858.

14. Mo L, Ma G, Dai C, Wang S, Li C, Ma T, Yang Z, He C, Xiao H, Wang L, Duan J, Zhang Y. Endovascular recanalization for symptomatic subacute and chronically occluded internal carotid artery: feasibility, safety, a modified radiographic classification system, and clinical outcomes. Neuroradiology. 2020;62:1323-34. 10.1007/s00234-020-02458-0.

15. Lin MS, Lin LC, Li HY, Lin CH, Chao CC, Hsu CN, Lin YH, Chen SC, Wu YW, Kao HL. Procedural safety and potential vascular complication of endovascular recanalization for chronic cervical internal carotid artery occlusion. Circ Cardiovasc Interv. 2008;1:119-25. 10.1161/CIRCINTERVENTIONS.108.772350.

16. Chen YH, Leong WS, Lin MS, Huang CC, Hung CS, Li HY, Chan KK, Yeh CF, Chiu MJ, Kao HL. Predictors for Successful Endovascular Intervention in Chronic Carotid Artery Total Occlusion. JACC 
Cardiovasc Interv. 2016;9:1825-32. 10.1016/j.jcin.2016.06.015.

17. Bouthillier A, van Loveren HR, Keller JT. Segments of the internal carotid artery: a new classification. Neurosurgery. 1996;38:425-32. 10.1097/00006123-199603000-00001. discussion 432-423.

18. Barber PA, Demchuk AM, Zhang J, Buchan AM. Validity and reliability of a quantitative computed tomography score in predicting outcome of hyperacute stroke before thrombolytic therapy. ASPECTS Study Group. Alberta Stroke Programme Early CT Score. Lancet. 2000;355:1670-4. 10.1016/s01406736(00)02237-6.

19. Higashida RT, Furlan AJ, Roberts H, Tomsick T, Connors B, Barr J, Dillon W, Warach S, Broderick J, Tilley B, Sacks D, Technology Assessment Committee of the American Society of I. Therapeutic N, Technology Assessment Committee of the Society of Interventional R (2003) Trial design and reporting standards for intra-arterial cerebral thrombolysis for acute ischemic stroke. Stroke 34:e109-37. 10.1161/01.STR.0000082721.62796.09.

20. Nahab F, Liu M, Rahman HA, Rangaraju S, Barrow D, Cawley CM, Grubb RL, Derdeyn CP, Adams HP, Videen TO, Zimmerman MB, Powers WJ. Recurrent Hemispheric Stroke Syndromes in Symptomatic Atherosclerotic Internal Carotid Artery Occlusions: The Carotid Occlusion Surgery Study Randomized Trial. Neurosurgery. 2020;87:137-41. 10.1093/neuros/nyz352.

21. Fan YL, Wan JQ, Zhou ZW, Chen L, Wang Y, Yao Q, Jiang JY. Neurocognitive improvement after carotid artery stenting in patients with chronic internal carotid artery occlusion: a prospective, controlled, single-center study. Vasc Endovascular Surg. 2014;48:305-10.

$10.1177 / 1538574414525863$.

\section{Figures}




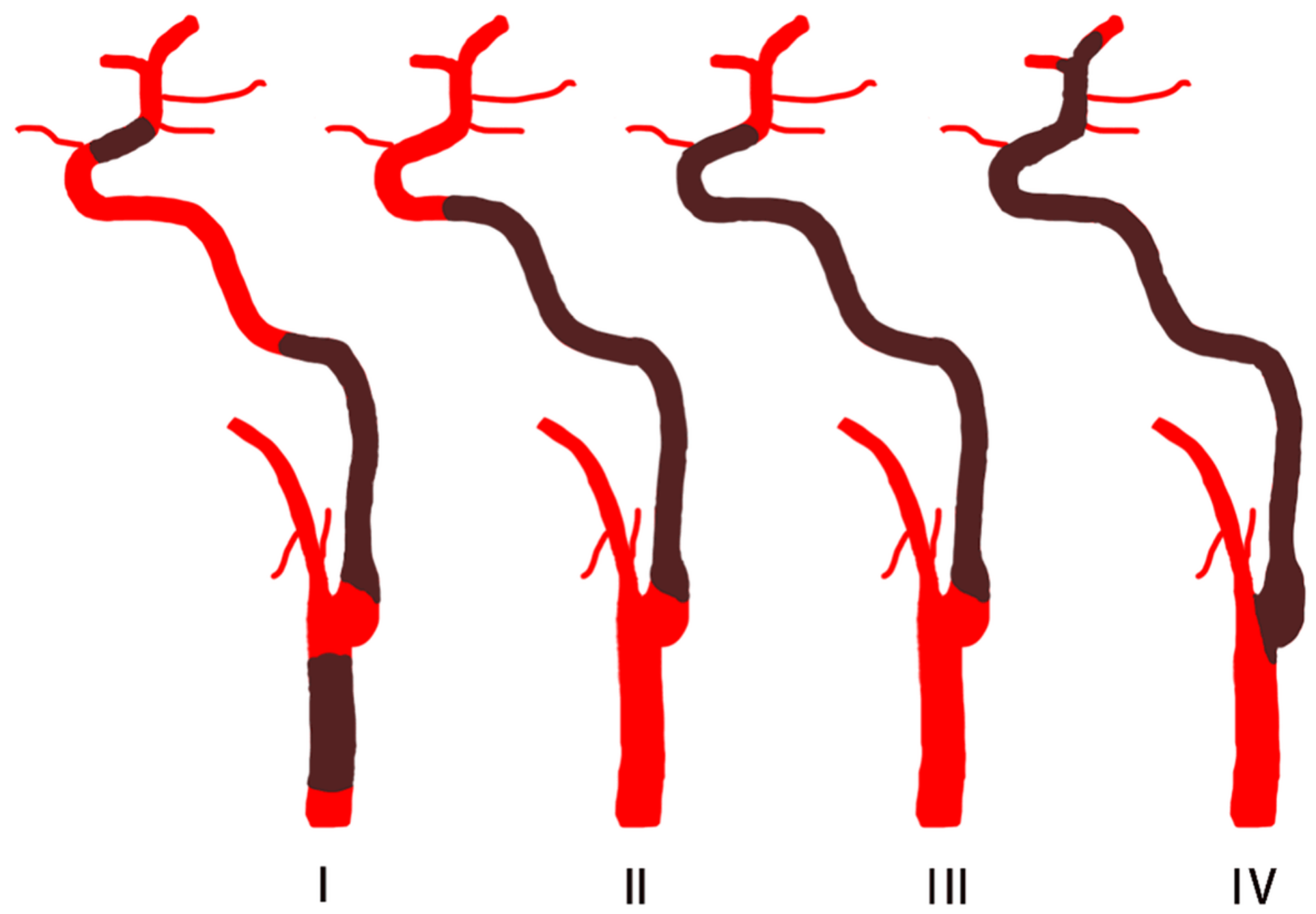

\section{Figure 1}

Schematic diagram of non-acute carotid artery occlusion (NACAO) typing. Type I: the occluded segment is located in the common carotid artery or involves $\leq 2$ consecutive segments in C1-C7. Type II: involving $\geq 3$ consecutive internal carotid artery segments proximal to C5. Type III: involving $\geq 3$ consecutive internal carotid artery segments, including C5. Type IV: the proximal end of the common carotid artery is unrecognizable, and the proximal or distal end of the internal carotid artery is unrecognizable. 

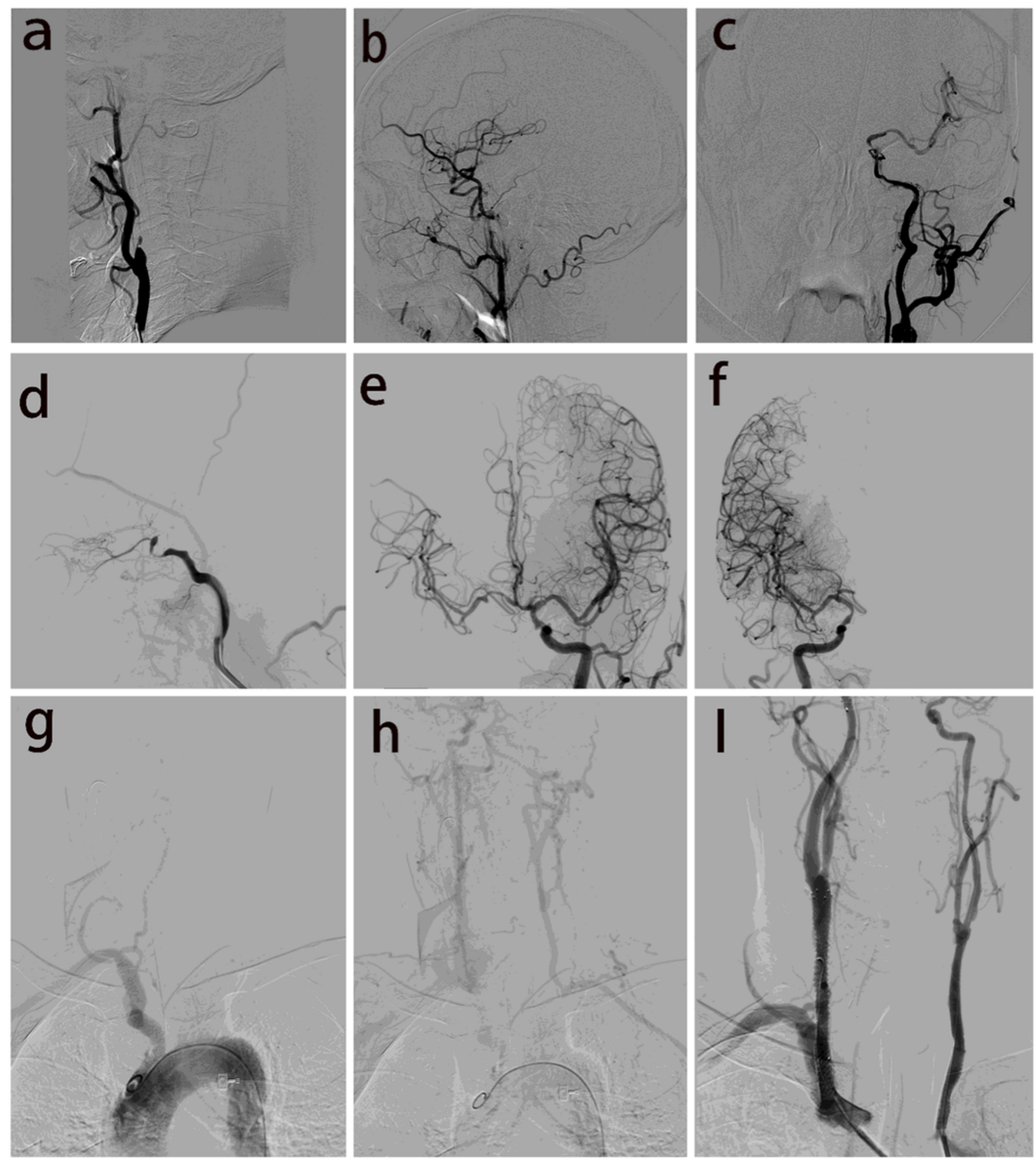

\section{Figure 2}

Endovascular Recanalization of Type I non-acute carotid artery occlusion (NACAO). C1-C2 occlusion (case 4, a-c). (a) Proximal end of the left internal carotid artery occlusion (C1). (b) Distal end of the occlusion (C2 horizontal part) compensated development through the ascending pharyngeal artery. (c) Successful recanalization ( $\mathrm{TICl} 2 \mathrm{~b}$ ). C6-C7 occlusion (case 23, d-f). (d) Right internal carotid artery occlusion proximal end (C6). (e) Occlusion distal end (C7) compensatory development through the 
anterior communicating artery. (f) Successful recanalization (thrombolysis in cerebral infarction (TICl) grade 3). Bilateral common carotid artery (CCA) occlusion (case 13, g-i). (g) Aortic arch angiography shows bilateral common carotid artery and left subclavian artery occlusion. (h) Late arterial compensatory development of bilateral common carotid artery distal ends through ascending carotid artery. (i) Bilateral CCA recanalization successfully ( $\mathrm{TICl}$ grade 3 ).

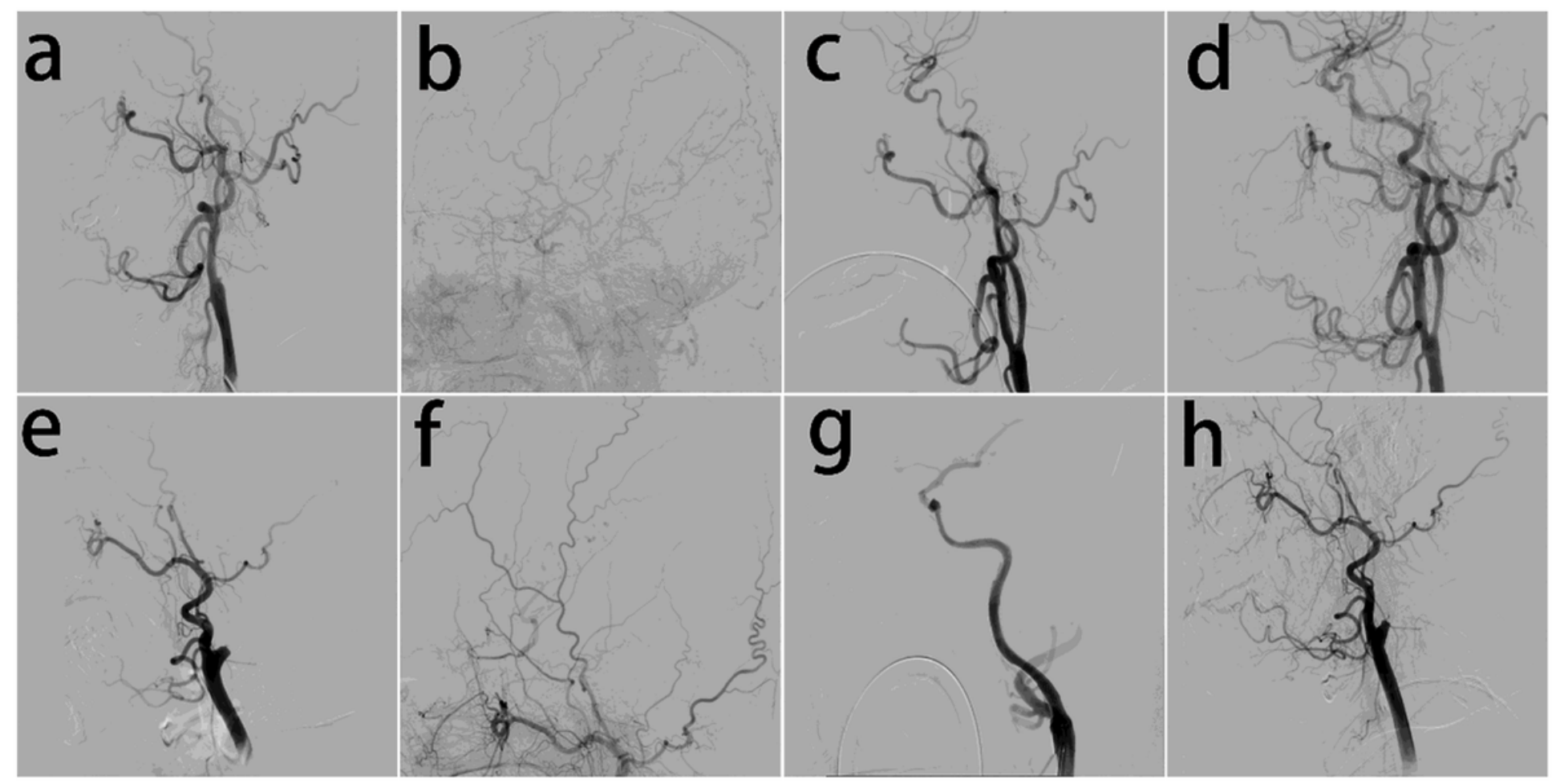

\section{Figure 3}

Endovascular recanalization of Types II and III non-acute carotid artery occlusion (NACAO). Type II C1-C4 occlusion (case 8, a-d). (a) Proximal end of the left internal carotid artery occlusion (C1). (b) Distal end of the occlusion (C4 horizontal segment) compensatory development through the ophthalmic artery. (c) Successful recanalization (thrombolysis in cerebral infarction (TICl) grade 3). (d) No restenosis after 4 months. Type III C1-C5 occlusion (case 16, e-h). (e) Proximal end of the left internal carotid artery occlusion (C1). (f) Distal end of the occlusion (C5) compensatory development through the ophthalmic artery. (g) Successful recanalization ( $\mathrm{TICl} 3$ ). (h) Re-occlusion after 10 months. 

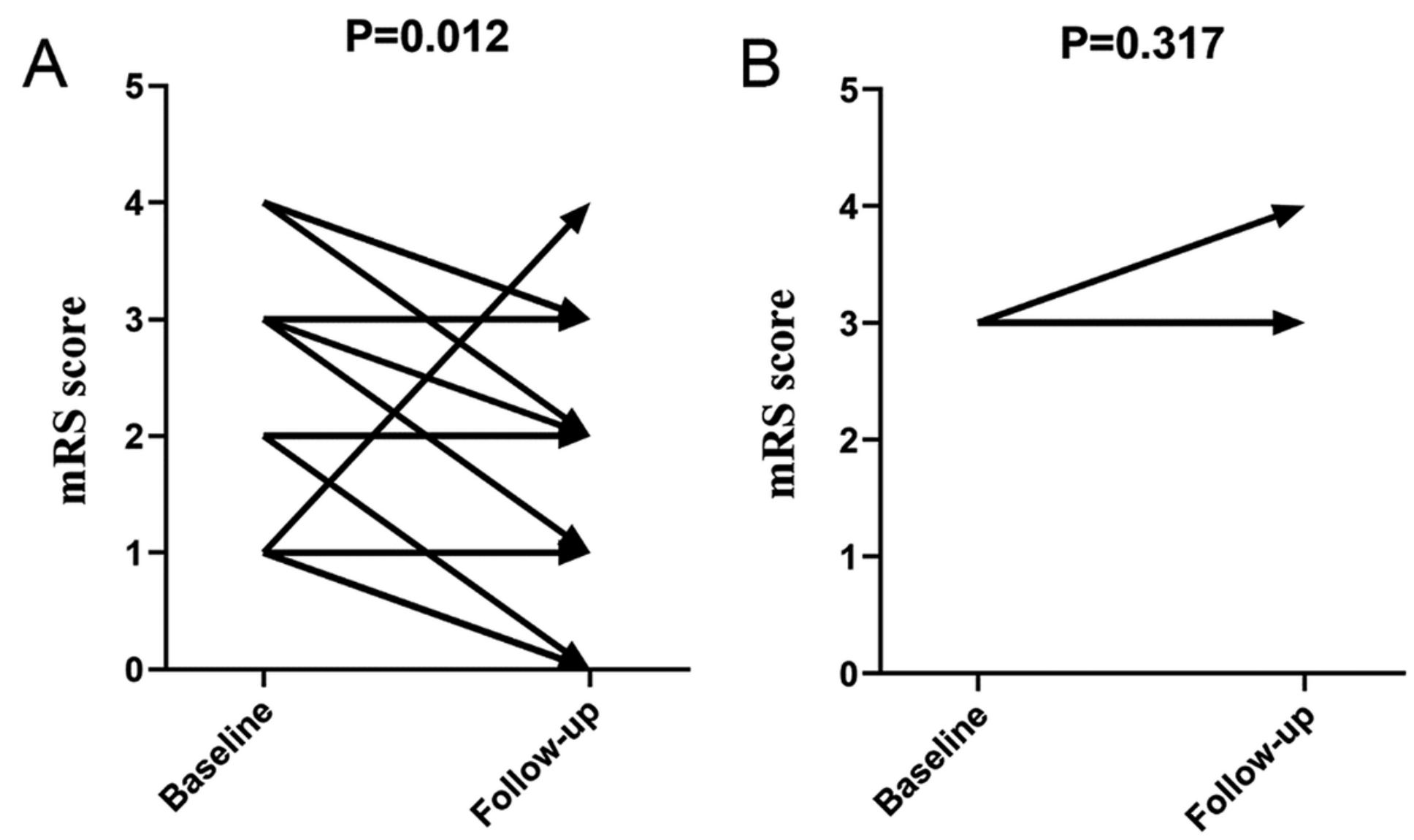

Figure 4

Comparison of the modified Rankin Scale (mRS) scores between preoperative and follow-up. (A) The average $\mathrm{mRS}$ score of the successful recanalization group (20 cases) at follow-up was lower than the average $m R S$ score before operation $(P=0.012)$. (B) There were no significant differences between the average follow-up mRS score and the average preoperative mRS score in the failure group (three cases) $(P=0.317)$.

\section{Supplementary Files}

This is a list of supplementary files associated with this preprint. Click to download.

- SupplementaryTableS1.docx 